\title{
Slow-light enhanced absorption for bio-chemical sensing applications: potential of low-contrast lossy materials
}

\section{Jesper Pedersen}

\section{Sanshui Xiao \\ $\underset{\text { asger@mailaps.org }}{\text { Niels Asger Mortensen }}$}

\begin{abstract}
MIC - Department of Micro and Nanotechnology, NanoDTU, Technical University of Denmark, DK2800 Kongens Lyngby, Denmark

MIC - Department of Micro and Nanotechnology, NanoDTU, Technical University of Denmark, DK2800 Kongens Lyngby, Denmark

MIC - Department of Micro and Nanotechnology, NanoDTU, Technical University of Denmark, DK2800 Kongens Lyngby, Denmark
\end{abstract}

Slow-light enhanced absorption in liquid-infiltrated photonic crystals has recently been proposed as a route to compensate for the reduced optical path in typical lab-on-a-chip systems for bio-chemical sensing applications. A simple perturbative expression has been applied to ideal structures composed of lossless dielectrics. In this work we study the enhancement in structures composed of lossy dielectrics such as a polymer. For this particular sensing application we find that the material loss has an unexpected limited drawback and surprisingly, it may even add to increase the bandwidth for low-index contrast systems such as polymer devices. [D0l: 10.2971/je0s.2008.08007]

Keywords: Biosensor, photonic crystal, optofluidics, slow light

\section{INTRODUCTION}

The strong emphasis on miniaturization of chemical analysis systems [1] has naturally fuelled a large effort in integrating optics and microfluidics in lab-on-a-chip microsystems [2, 3] and more recently the emerging field of optofluidics [4]-[6] has increased this interest further.

The miniaturization of chemical analysis systems has created a new paradigm with a broad variety of phenomena, properties, and applications benefitting from the down-scaling in size [1]. However, the case of optical sensing and detection remains an exception because the light-matter interactions suffer from the reduced optical path length $L$ in lab-on-achip systems compared to their macroscopic counterparts. For Beer-Lambert absorption measurements, a typical size reduction by two orders of magnitude will penalize the optical sensitivity in an inversely proportional manner as has been demonstrated by Mogensen et al. [7].

In a simple picture, the above problem stems from the reduced light-matter interaction time $\tau$. For a homogeneous system of length $L$, such as a classical absorbance cell, we have the following Wigner-Smith delay time

$$
\tau \sim L / c,
$$

with $c$ being the speed of light. Obviously, for small systems the photons spend too little time inside the sample and thus do not inherit strong fingerprints from the chemical species they were supposed to interact with. As a result, only very high concentrations can be quantified compared to the levels that can be detected with a macroscopic optical path length. On the other hand, lab-on-a-chip systems are often envisioned for applications dealing with quite minute sample volumes with very low concentrations of bio-molecules. The use of slow-light phenomena in liquid-infiltrated Bragg stacks and photonic crystals, see Figure 1, has recently been proposed as a way to deal with this conflict of length scales $[8,9]$.

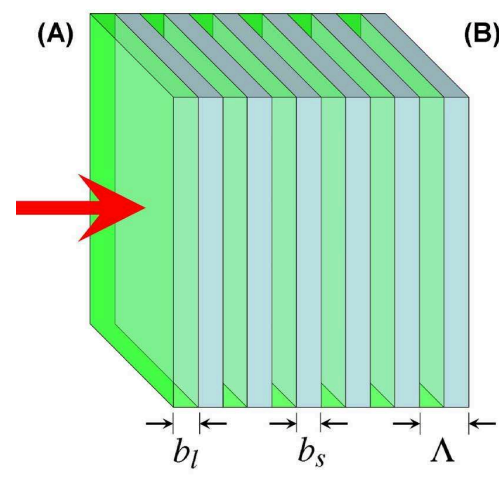

B)

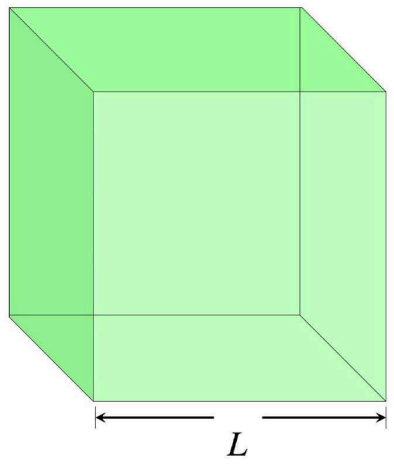

FIC. 1 (A) Liquid-infiltrated Bragg stack composed of alternating solid and liquid layers of thickness $b_{s}$ and $b_{l}$, respectively. (B) Schematic illustration of a corresponding classical Beer-Lambert absorbance cell where the incident light passes through a spatially homogeneous medium, i.e. the liquid sample to be analyzed.

In the context of Beer-Lambert absorption, the modulation of the transmitted intensity is typically expressed as

$$
I=I_{0} \exp (-\alpha L)=I_{0} \exp \left(-\gamma \alpha_{l} L\right)
$$

where $\alpha_{l}$ is the absorption coefficient for the absorbing liquid and $I_{0}$ is the transmitted intensity with a vanishing concentration of the absorbing bio-chemical species in the liquid, while $I$ is then the transmitted intensity at a finite concentration which is to be quantified by the absorbance measurement. To lowest order in the absorption, the dimensionless enhancement factor $\gamma \equiv \alpha / \alpha_{l}$ can be expressed as [8]

$$
\gamma=f \times \frac{c / n_{l}}{v_{g}},
$$


where $0<f<1$ is a dimensionless number quantifying the relative optical overlap with the liquid [10]. The fraction on the right-hand side expresses the ratio of the group velocity $c / n_{l}$ in the bare liquid to the group velocity $v_{g}$ in the liquidinfiltrated structure, thus clearly illustrating the enhancement by slow-light propagation $\left(v_{g} \ll c\right)$. The above perturbative expression has been derived by standard first-order electromagnetic perturbation theory [10] as well as by a scattering matrix approach combined with the concept of the WignerSmith delay time [11]. So far, the concept has only been illustrated by means of simulations of ideal structures comprising lossless dielectric materials infiltrated by the absorbing bioliquid of interest. In this paper we illustrate the effect for lossy materials by means of analytical results for a Bragg stack composed of a lossy dielectric such as typical bio-compatible polymer materials envisioned to play an important role for future low-cost disposable lab-on-a-chip systems.

\section{THE BRAGG STACK EXAMPLE}

The Bragg stack serves as an illustrative example where the dispersion relation can be obtained by analytical means and where band diagrams are available also in the case of absorbing materials. The structure is illustrated in Figure 1 and is composed of alternating layers of thickness $b_{l}$ and $b_{s}$ with $\Lambda=b_{l}+b_{s}$ being the pitch and $\epsilon_{l}=\epsilon_{l}^{\prime}+i \epsilon_{l}^{\prime \prime} \simeq n_{l}^{2}+i n_{l} \alpha_{l} / k$ and $\epsilon_{s}=\epsilon_{s}^{\prime}+i \epsilon_{s}^{\prime \prime} \simeq n_{s}^{2}+i n_{s} \alpha_{s} / k$ being the corresponding relative dielectric permittivities of the liquid and the solid. The absorption coefficients $\alpha_{l}$ and $\alpha_{s}$ are defined such that to lowest order in $\alpha$ the intensity decays exponentially, $I(z) \propto$ $|\exp (i \sqrt{\epsilon} k z)|^{2}=\exp (-\alpha z)$, in the corresponding spatially homogenous materials.

\subsection{Dispersion relation}

In the following we follow a recent derivation by Kim et al. [12] where the dispersion relation is given by the root of the following equation

$$
\cos (\kappa \Lambda)=F(k),
$$

where $\kappa$ is the Bloch wave vector and $k(\omega)=\omega / c$ is the freespace wave vector. The dimensionless function on the righthand side is given by

$$
\begin{aligned}
F(k) & =\cos \left(\sqrt{\epsilon_{l}} k b_{l}\right) \cos \left(\sqrt{\epsilon_{s}} k b_{s}\right) \\
& -\frac{\epsilon_{l}+\epsilon_{s}}{2 \sqrt{\epsilon_{l}} \sqrt{\epsilon_{s}}} \sin \left(\sqrt{\epsilon_{l}} k b_{l}\right) \sin \left(\sqrt{\epsilon_{s}} k b_{s}\right)
\end{aligned}
$$

which is symmetric under the exchange of $\left(\varepsilon_{l}, b_{l}\right)$ and $\left(\varepsilon_{s}, b_{s}\right)$.

The group velocity can be found explicitly by noting that $v_{g}=$ $\partial \omega / \partial \kappa=c \partial k / \partial \kappa$ from which we get

$$
\left(\frac{v_{g}}{c}\right)^{-1}=\frac{1}{\Lambda} \frac{\partial}{\partial k} \arccos \{F(k)\}=\frac{1}{\Lambda} \frac{-\frac{\partial}{\partial k} F(k)}{\sqrt{1-F^{2}(k)}},
$$

where it is implicitly assumed that the real part is taken.

In panel (A) of Figure 2 we illustrate the typical band structure by means of an example with alternating layers of air

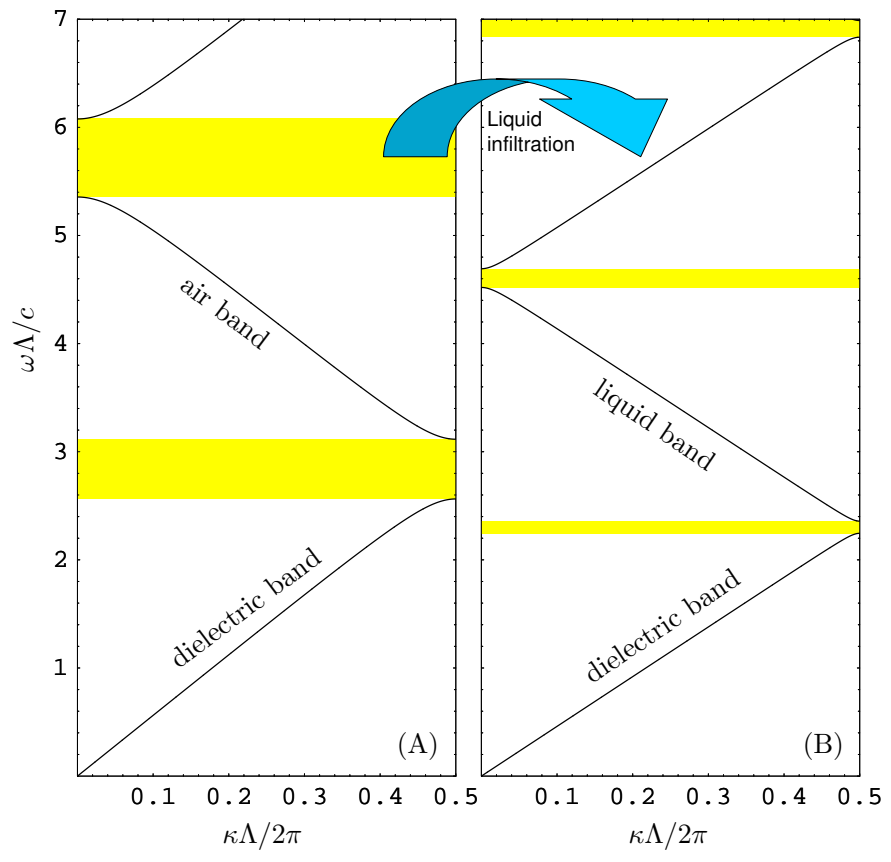

FIG. 2 (A) Band diagram for a Bragg stack with $n_{l}=1$ (corresponding to air), $n_{s}=1.5$ (corresponding to a typical polymer), $b_{l}=0.8 \Lambda$, and $b_{s}=0.2 \Lambda$. (B) Band diagram for the structure in $(\mathrm{A})$, but with the air regions infiltrated by a liquid with $n_{l}=1.33$.

(with $n_{l}=1$ and $b_{l}=0.8 \Lambda$ ) and a typical polymer material (with $n_{s}=1.5$ and $b_{s}=0.2 \Lambda$ ). Polymers are often considered to provide too low an index contrast for the formation of pronounced photonic band gaps. However, slow-light phenomena in polymer systems are still feasible as experimentally demonstrated for e.g. photonic band-edge lasing [13]. When infiltrating the structure by a typical biological liquid $\left(n_{l}=1.33\right)$ the index contrast is decreased even further and band gaps are expected to narrow. Panel (B) illustrates this for the same structure as in panel (A), but with the air regions infiltrated by a liquid with $n_{l}=1.33$. In general bands shift down in frequency, but it is noteworthy that some bands tend to have a more pronounced shift than others. This has lead to a classification of bands in terms of liquid and dielectric bands [10] similarly to the classification of dielectric and air bands employed widely in the photonic crystal community [14]. Obviously, the air (liquid) band is most susceptible to the infiltration by the liquid as seen by the pronounced downshift in frequency, while the dielectric band is less perturbed when introducing the liquid in the air regions. As we shall see, this different nature of the bands also becomes important for the impact of loss in the solid layers where the electrical field tends to localize for the dielectric band.

\subsection{The Beer-Lambert enhancement factor}

In order to calculate the enhancement factor $\gamma$ explicitly we consider the general case of a complex valued Bloch wave vector $\kappa=\kappa^{\prime}+i \kappa^{\prime \prime}$ with the prime and double-prime indicating the real and imaginary part, respectively. Taylor expanding the left-hand side of Eq. (4) to lowest order in $\kappa^{\prime \prime}$ and likewise the right-hand side to lowest order in the liquid absorption 
parameter $\alpha_{l}$ we get

$$
\gamma \equiv \frac{2 \kappa^{\prime \prime}}{\alpha_{l}}=\left.\frac{2}{i \Lambda} \frac{\frac{\partial}{\partial \alpha_{l}} F(k)}{\sqrt{1-F^{2}(k)}}\right|_{\alpha_{l}=0} .
$$

This result can be shown to be fully consistent with the general expression in Eq. (3).

In Figure 3 we illustrate the enhancement for the example with $n_{l}=1.33, n_{s}=1.5, b_{l}=0.8 \Lambda$, and $b_{s}=0.2 \Lambda$, corresponding to the band structure shown in panel (B) of Figure 2 . We focus on the first band gap centered around $\omega \Lambda / c \simeq 2.3$,

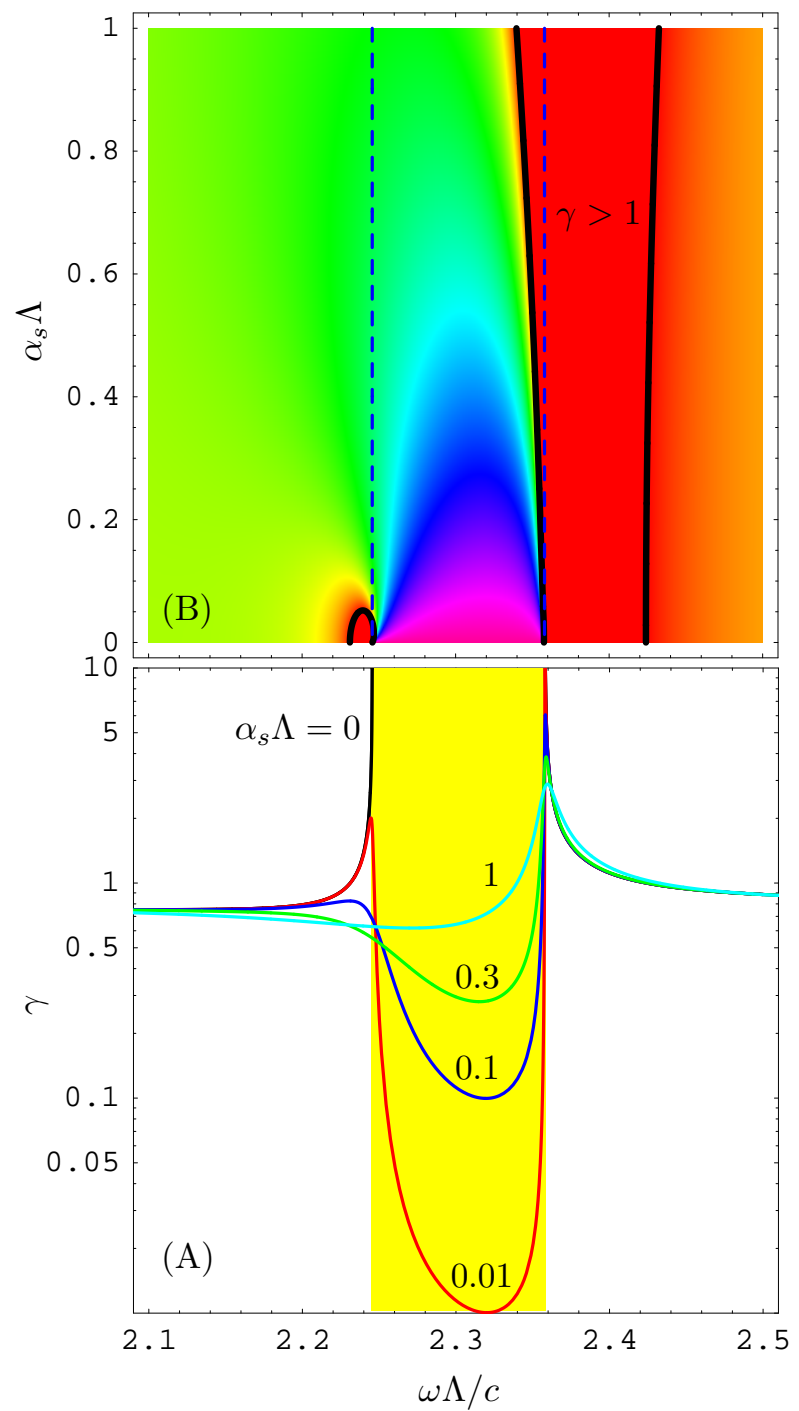

FIG. 3 Enhancement of liquid absorption for a Bragg stack with $n_{l}=1.33, n_{s}=$ $1.5, b_{l}=0.8 \Lambda$, and $b_{s}=0.2 \Lambda$. (A) Beer-Lambert enhancement factor $\gamma$ versus normalized frequency $\omega \Lambda / c$ around the first band gap (indicated by yellow shading) for varying values of the absorption $\alpha_{S} \Lambda$ in the solid layers. (B) The Beer-Lambert enhancement factor $\gamma$ in a $\alpha_{s} \Lambda$ versus $\omega \Lambda / c$ plot. Regions with $\gamma>1$ are shown in red and the black solid lines show the contour for $\gamma=1$ while the dashed blue lines indicate the band-gap edges for the lossless structure.

where the band below the band gap is a dielectric band, while the band just above the band gap is a liquid band. For the solid layers we include a finite damping quantified by a varying $\alpha_{s}$. From the classification of the bands we expect that the loss will broaden the enhancement in an asymmetric manner with respect to the center of the band gap, such that the dielectric band is most susceptible to an increasing $\alpha_{s}$. Indeed, panel (A) reveals exactly such a behavior, with a pronounced smearing of the singularity in $\gamma$ at the lower band edge, while the singularity at the upper band edge sustains the damping to a higher degree. This picture is in full agreement with the experimental observations of enhanced red-absorption in dyedoped inverse-opal photonic crystals by Nishimura et al. [15]. The plot of $\gamma$ in the $\alpha_{s}$ versus $\omega$ diagram in panel (B) shows this in more detail. The solid black lines inclose regions with $\gamma>1$ where the liquid-infiltrated structure is superior to its classical counterpart without any microstructure. Interestingly, damping in the solid material is seen not to be a serious limitation when working with the liquid band. In fact the damping may even help increase the bandwidth slightly of the otherwise quite narrow singularity, while still allowing for a significant enhancement, perhaps even by one order of magnitude.

\section{CONCLUSION}

An optofluidic slow-light phenomenon has recently been proposed as a mechanism allowing for enhanced absorption measurements in lab-on-a-chip systems with a reduced optical path compared to their macroscopic counterparts. In this work we have explored the use of lossy dielectric materials such as polymer which is typically the preferred class of materials for lab-on-a-chip systems. By means of the Bragg stack example we have explicitly shown that the enhancement does not critically depend on the polymer loss and somewhat surprisingly the polymer loss may even serve as a desired broadening mechanism allowing for a higher measurement bandwidth.

\section{ACKNOWLEDGEMENTS}

This work is financially supported by the Danish Council for Strategic Research through the Strategic Program for Young Researchers (grant no: 2117-05-0037) as well as the Danish Research Council for Technology and Production Sciences (grants no: 27407-0080 \& 274-07-0379).

\section{References}

[1] D. Janasek, J. Franzke, and A. Manz, "Scaling and the design of miniaturized chemical-analysis systems" Nature $442,374-380$ (2006).

[2] E. Verpoorte, "Chip vision - optics for microchips", Lab Chip 3, $42 \mathrm{~N}-52 \mathrm{~N}(2003)$.

[3] K. B. Mogensen, H. Klank, and J. P. Kutter, "Recent developments in detection for microfluidic systems", Electrophoresis 25, 34983512 (2004).

[4] D. Psaltis, S. R. Quake, and C. H. Yang, "Developing optofluidic technology through the fusion of microfluidics and optics", Nature 442, 381-386 (2006).

[5] C. Monat, P. Domachuk, and B. J. Eggleton, "Integrated optofluidics: A new river of light", Nature Photonics 1, 106-114 (2007).

[6] D. Erickson, "Special issue on "Optofluidics"”, Microfluid. Nanofluid. 4, 1 (2008). 
[7] K. B. Mogensen, J. El-Ali, A. Wolff, and J. P. Kutter, “Integration of polymer waveguides for optical detection in microfabricated chemical analysis systems", Appl. Optics 42, 4072-4079 (2003).

[8] N. A. Mortensen and S. Xiao, "Slow-light enhancement of BeerLambert-Bouguer absorption", Appl. Phys. Lett. 90, 141108 (2007).

[9] J. Pedersen and N. A. Mortensen, "Enhanced circular dichroism via slow light in dispersive structured media", Appl. Phys. Lett. 91, 213501 (2007).

[10] N. A. Mortensen, S. Xiao, and J. Pedersen, "Liquid-infiltrated photonic crystals - enhanced light-matter interactions for lab-on-achip applications", Microfluid. Nanofluid. 4, 117 (2008).

[11] M. E. V. Pedersen, L. S. Rishøj, H. Steffensen, S. Xiao, and N. A. Mortensen, "Slow-light enhanced optical detection in liquidinfiltrated photonic crystals", 0pt. Quant. Electron. 39, 903 (2007).
[12] K. Kim, H. Yoo, D. H. Lee, and H. Lim, "Exact analytical expressions for the dispersion relation of one-dimensional chiral photonic crystals", Waves Random Complex Media 16, 75-84 (2006).

[13] F. B. Arango, M. B. Christiansen, M. Gersborg-Hansen, and A. Kristensen, "Optofluidic tuning of photonic crystal band edge lasers", Appl. Phys. Lett. 91, 223503 (2007)

[14] J. D. Joannopoulos, R. D. Meade, and J. N. Winn, Photonic crystals: molding the flow of light (Princeton University Press, Princeton, 1995).

[15] S. Nishimura, N. Abrams, B. A. Lewis, L. I. Halaoui, T. E. Mallouk, K. D. Benkstein, J. van de Lagemaat, and A. J. Frank, "Standing wave enhancement of red absorbance and photocurrent in dyesensitized titanium dioxide photoelectrodes coupled to photonic crystals", J. Am. Chem. Soc. 125, 6306-6310 (2003). 